\title{
Delicate but significant changes in blood pressure-the importance of clinical research by frontline clinicians
}

\author{
Kei Asayama ${ }^{1,2,3}$
}

Received: 11 January 2022 / Accepted: 18 January 2022 / Published online: 10 February 2022

(c) The Author(s), under exclusive licence to The Japanese Society of Hypertension 2022

A meta-analysis of trials demonstrated that the blood pressure gradient between antihypertensive drug intervention groups largely accounts for most differences in cardiovascular outcome [1]. A 4-mmHg decrease in average systolic blood pressure in Japanese men and women is estimated to reduce stroke mortality by 8.9 and $5.8 \%$, respectively [2]. Although intensive blood pressure control is essential, more than $50 \%$ of Japanese patients who received antihypertensive medication still had a blood pressure of $\geq 140$ / $\geq 90 \mathrm{mmHg}$ irrespective of their age [3]. Therefore, small changes in blood pressure should be detected without overlooking and be assessed appropriately to avoid clinical inertia and construct future treatment strategies.

The behaviors of patients have been modified by the COVID-19 pandemic and related events, e.g., by increasing intervals between medical visits [4] and promoting telemonitoring of blood pressure [5]. It should be noted that the largest COVID-19 cluster outside China for the period February 7-24, 2020, occurred on the cruise ship "the Diamond Princess" anchored and quarantined at Yokohama port, located in Kanagawa prefecture, Japan, and the cluster attracted global attention [6]. Moreover, a month before the Diamond Princess quarantine, media news worried about the potential impact of Chinese New Year (spring festival) during January 24-30, 2020 on the COVID-19 pandemic in Japan. Affected by these social situations, individuals' state of mind and body condition during the first quarter of 2020

Kei Asayama

kei@asayama.org

1 Department of Hygiene and Public Health, Teikyo University School of Medicine, Tokyo, Japan

2 Studies Coordinating Centre, Research Unit Hypertension and Cardiovascular Epidemiology, KU Leuven Department of Cardiovascular Sciences, University of Leuven, Leuven, Belgium

3 Tohoku Institute for Management of Blood Pressure, Sendai, Japan would be different from those in previous years, resulting in changes in blood pressure and inevitably in future cardiovascular risk.

Kobayashi et al. reported changes in conventional office and self-measured home blood pressures among patients before and during the COVID-19 pandemic based on a clinical observational study conducted by practitioners in Sagamihara, Kanagawa prefecture, which was the suburb of the Tokyo metropolitan area [7]. Of the 919 assessed patients, 748 were set as the study population to be analyzed. The first catchment periods of blood pressure were from April to June 2019 ( $n=567$ who measured office blood pressure and 347 of those who measured home blood pressure), which should be called the baseline period because COVID-19 did not emerge during that period. The second period was January to March 2020 (as the harbinger period; $n=748$, and 535 had home blood pressure), and the third period was from April 7 to May 31, 2020, in which the first state of emergency in Japan had been declared (as the emergency period; the same number as those in the harbinger period). The emergency period was treated as the main period in the analysis. Because the seasons coincided, the effect of seasonal blood pressure variation [8] was minimal on the comparison between the baseline and emergency periods, and the mean blood pressure differences were $4.1 /-1.4$ and $-2.0 /-0.6 \mathrm{mmHg}$ in office $(n=567)$ and at home $(n=347)$ measurements, respectively. In the same population, the difference in blood pressures between the baseline and harbinger periods was $1.3 / 0.7 \mathrm{mmHg}$ in office and $-0.7 / 0.1 \mathrm{mmHg}$ at home measurements. The primary comparison was performed among 748 and 535 participants who measured their blood pressure at the office or home, respectively, during the harbinger and emergency periods $(2.1 / 0.8 \mathrm{mmHg}$ in office and $-1.3 /-0.6 \mathrm{mmHg}$ at home measurements; $P \leq 0.03$ ). However, the direct comparison of office and home blood pressures was restricted since the study set different patients for each measurement. Furthermore, the different study populations per period made the findings difficult to compare directly. Although the results in 
this study were essentially similar regardless of the number of analyzed patients, setting up the study population sometimes affects the results and therefore should be considered carefully. The definition of the study population is also an essential checkpoint for followers who use the current outputs in a meta-analysis.

The study group further collected a questionnaire regarding the change in stress by the declaration of emergency among the same 748 patients (the month and date were not given) [7]. Unfortunately, very few clinically meaningful results were reported. For instance, in the receiver operating characteristic analysis of the cutoff value of the total stress for an increase in mean arterial office blood pressure, the area under the curve was 0.54 , and $95 \%$ confidence intervals crossed 0.5 (0.49-0.58).

The opposite shift of office and home blood pressures resulted in the increase of patients with hypertension accompanied by the white-coat effect [2] or office uncontrolled hypertension [9], from $13 \%$ in the harbinger period to $17 \%$ in the emergency period. They are usually called patients with white-coat hypertension, while the term is originally used for untreated people because antihypertensive drug agents extensively modify blood pressure. In the International Database of HOme blood pressure in relation to Cardiovascular Outcomes, whitecoat (office uncontrolled) hypertension assessed by home blood pressure measurements was a significant cardiovascular risk factor in untreated people but not in treated patients [10]. This discrepancy can be explained by the effective antihypertensive treatment based on their elevated office blood pressure [10]. Furthermore, people with white-coat hypertension have a greater risk for sustained hypertension than normotension, i.e., their out-of-office blood pressure increases at the later stage [11]. It was also reported among treated patients, i.e., compared with patients with controlled hypertension, office uncontrolled hypertension was associated with a 2.2 times higher risk of developing sustained hypertension at 1 year [9]. A longterm follow-up of the study patients, preferably with sufficient data on drug regime, will provide further insight into the impact of the white-coat effect and the long-term trend of office and home blood pressures on cardiovascular complications.

Although there were several potential limitations, the study by Kobayashi et al. [7] drove research activities in Japan, which may be why the reviewers thought highly of the results of this study. Unlike other countries, the number of noncommercial clinical studies conducted in Japan began to decrease in 2018 [12]. This undesirable trend reflected the implementation of the Clinical Trials Act (CTA), which was established in the same year [12]. Although CTA was introduced to improve the quality and transparency of clinical trials in Japan, the burden of CTA implementation includes an increasing cost of the review board and insurance for clinical trials and complicated administrative processes, including setup for new research and minor changes to protocols [13]. Although the decrease in the study number due to CTA might be a temporary trend [13], the increasing burden is also relevant for implementing other research ethics guidelines.

Nevertheless, researchers need to keep in mind that stricter rules are a part of a global effort to eliminate research misconduct and are the public demand to improve research quality. Therefore, following the ethical guidelines is a minimum requirement and shall be addressed appropriately; if one finds it difficult, one can consult an expert. Given that hypertension is the leading contributor to cardiovascular complications, more research outputs, particularly regarding hypertension conducted in frontline clinical practice, are expected.

\section{Compliance with ethical standards}

Conflict of interest KA received research support from Omron Healthcare and honoraria from Takeda Pharmaceutical.

Publisher's note Springer Nature remains neutral with regard to jurisdictional claims in published maps and institutional affiliations.

\section{References}

1. Staessen JA, Wang JG, Thijs L. Cardiovascular protection and blood pressure reduction: a meta-analysis. Lancet. 2001;358: $1305-15$.

2. Umemura S, Arima H, Arima S, Asayama K, Dohi Y, Hirooka Y, et al. The Japanese Society of Hypertension guidelines for the management of hypertension (JSH 2019). Hypertens Res. 2019;42:1235-481.

3. Asayama K, Kinoshita Y, Watanabe S, Ohkubo T, Ando T, Harada A, et al. Impact of diastolic blood pressure threshold for the young population: the Japan Arteriosclerosis Longitudinal Study (JALS). J Hypertens. 2019;37:652-3.

4. Powis M, Milley-Daigle C, Hack S, Alibhai S, Singh S, Krzyzanowska MK. Impact of the early phase of the COVID pandemic on cancer treatment delivery and the quality of cancer care: a scoping review and conceptual model. Int $\mathrm{J}$ Qual Health Care. 2021;33:mzab088.

5. Park SH, Shin JH, Park J, Choi WS. An updated meta-analysis of remote blood pressure monitoring in urban-dwelling patients with hypertension. Int J Environ Res. Public Health. 2021;18:10583.

6. Jimi H, Hashimoto G. Challenges of COVID-19 outbreak on the cruise ship Diamond Princess docked at Yokohama, Japan: a realworld story. Glob Health Med. 2020;2:63-65.

7. Kobayashi K, Chin K, Umezawa S, Ito S, Yamamoto H, Nakano $\mathrm{S}$, et al. Influence of stress induced by the first announced state of emergency announced due to coronavirus disease 2019 on outpatient blood pressure management in Japan. Hypertens Res. 2021. https://doi.org/10.1038/s41440-021-00832-w.

8. Hanazawa T, Asayama K, Watabe D, Hosaka M, Satoh M, Yasui $\mathrm{D}$, et al. Seasonal variation in self-measured home blood pressure among patients on antihypertensive medications: HOMED-BP study. Hypertens Res. 2017;40:284-90. 
9. Cacciolati C, Hanon O, Dufouil C, Alperovitch A, Tzourio C. Categories of hypertension in the elderly and their 1-year evolution. The Three-City Study. J Hypertens. 2013;31:680-9.

10. Stergiou GS, Asayama K, Thijs L, Kollias A, Niiranen TJ, Hozawa A, et al. Prognosis of white-coat and masked hypertension: International Database of HOme blood pressure in relation to Cardiovascular Outcome. Hypertension. 2014;63:675-82.

11. Ugajin T, Hozawa A, Ohkubo T, Asayama K, Kikuya M, Obara $\mathrm{T}$, et al. White-coat hypertension as a risk factor for the development of home hypertension: the Ohasama study. Arch Intern Med. 2005;165:1541-6.

12. Tsutsumi I, Tsutsumi Y, Yoshida C, Komeno T, Imanaka Y. Impact of the Clinical Trials Act on noncommercial clinical research in Japan: an interrupted time-series analysis. J Epidemiol. 2022;32:27-33.

13. Ito Y, Yamamoto S, Nakamura K. What has happened since the implementation of the Clinical Trials Act? Epidemiologists need to know. J Epidemiol. 2022;32:2-3. 